\title{
Erratum to: Rheumatology training experience across Europe: analysis of core competences
}

Francisca Sivera ${ }^{1 *}$, Sofia Ramiro², Nada Cikes ${ }^{3}$, Maurizio Cutolo ${ }^{4}$, Maxime Dougados ${ }^{5}$, Laure Gossec ${ }^{6}$, Tore K. Kvien? Ingrid E. Lundberg ${ }^{8,9}$, Peter Mandl ${ }^{10}$, Arumugam Moorthy ${ }^{11}$, Sonia Panchal ${ }^{12}$, José A. P. da Silva ${ }^{13}$, Johannes W. Bijlsma ${ }^{14}$ and the Working Group on Training in Rheumatology across Europe

\section{Erratum}

Unfortunately, after publication of this article [1], it was noticed that the affiliation for Peter Mandl was incorrect. The correct affiliation is 'Divison of Rheumatology, Medical University of Vienna, Vienna, Austria'. This can be seen in the corrected author details below.

\begin{abstract}
Author details
'Department Reumatologia, Hospital General Universitario de Elda, ctra Sax s/n, Elda, Alicante 03600, Spain. '2Leiden University Medical Center, Leiden, The Netherlands. ${ }^{3}$ University of Zagreb School of Medicine, University Hospital Centre, Zagreb, Croatia. ${ }^{4}$ Research Laboratories and Academic Division of Clinical Rheumatology, Postgraduate School on Rheumatology, Department of Internal Medicine University of Genova, Genova, Italy. ${ }^{5}$ Université Paris Descartes University, Department of Rheumatology, Hôpital Cochin, Assistance Publique-Hôpitaux de Paris; INSERM (U1153):

Epidemiologie Clinique et Biostatistiques, PRES Sorbonne Paris-Cité, Paris, France. ${ }^{6}$ Sorbonne Universités, UPMC Univ Paris 06, Institut Pierre Louis d'Epidémiologie et de Santé Publique; AP-HP, Pitié Salpêtrière Hospital, Department of Rheumatology, F-75013 Paris, France. ${ }^{7}$ Department of Rheumatology, Diakonhjemmet Hospital, Oslo, Norway. ${ }^{8}$ Rheumatology Unit, Department of Medicine, Karolinska University Hospital, Solna, Sweden. ${ }^{9}$ Karolinska Institutet, Stockholm, Sweden. ${ }^{10}$ Divison of Rheumatology, Medical University of Vienna, Vienna, Austria. ${ }^{11}$ University Hospitals of Leicester, Leicester, UK. ${ }^{12}$ University Hospitals of Leicester NHS Trust, Leicester, UK. ${ }^{13}$ Centro Hospitalar e Universitário de Coimbra, Faculdade de Medicina da Universidade de Coimbra, Coimbra, Portugal. ${ }^{14}$ University Medical Center Utrecht, Utrecht, The Netherlands.
\end{abstract}

Received: 29 November 2016 Accepted: 30 November 2016 Published online: 19 December 2016

\section{Reference}

1. Sivera F, Ramiro S, Cikes N, et al. Rheumatology training experience across Europe: analysis of core competences. Arthritis Res Ther. 2016;18:213. doi:10.1186/s13075-016-1114-y.

\footnotetext{
* Correspondence: fransimas@yahoo.es

'Department Reumatologia, Hospital General Universitario de Elda, ctra Sax

s/n, Elda, Alicante 03600, Spain
} 